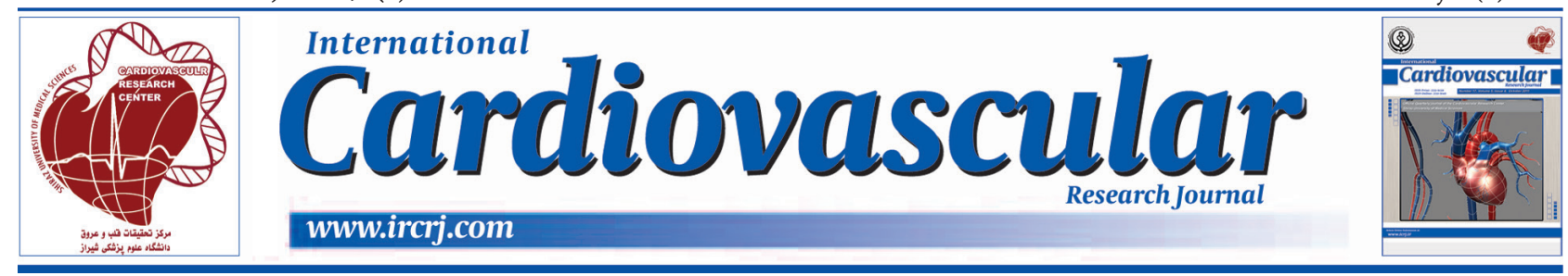

\title{
Comparison of US-Guided Catheterization of the Right Internal Jugular Vein Using Medial-Oblique and Short Axis Techniques
}

\author{
Hamid Kamalipour, ${ }^{1}$ Shahrbano Shahbazi, ${ }^{2}$ Mohammad Mehdi Derakhshan,, ${ }^{2,}$ Mohammad Taghi \\ Moinvaziri, ${ }^{2}$ and Elaheh Allahyari ${ }^{2}$
}

${ }^{1}$ Anesthesia Department, Ordibehesht Hospital, Shiraz, IR Iran

${ }^{2}$ Shiraz Anesthesiology and Critical Care Research Center, Shiraz University of Medical Sciences, Shiraz, IR Iran

\begin{tabular}{l}
\hline A R T I C L E I N F O \\
\hline Article Type: \\
Research Article \\
\hline
\end{tabular}

Article History:

Received: 15 Aug 2014

Revised: 31 May 2015

Accepted: 14 Jun 2015

\section{Keywords:}

Ultrasonography

Catheterization

Central Venous Catheter

\begin{abstract}
A B S T R A C T
Background: Although some investigations have shown higher rates of successful first attempt and fewer attempts by using ultrasound-guided Internal Jugular Vein (IJV) catheterization, arterial puncture is still common.

Objectives: The present study aimed to investigate US-guided catheterization of the right IJV via medial-oblique technique and also compare this technique to short-axis technique in open-heart surgery patients.

Patients and Methods: In this randomized clinical trial, 80 patients referred to cardiac operating room of Namazi hospital, Shiraz, Iran from March to July 2014 were selected using census method. Block randomization with website was also done. Then, the patients were divided into two groups of 40, Short Axis Group (SAG) and Medial-Oblique Group (M-OG). For short-axis technique, patient's head was positioned at zero degree angulation with his trunk. For medial-oblique technique, on the other hand, patient's head was tilted to left to 45 degrees between the head and trunk. Sex, age, Body Mass Index (BMI), access time, guidewire time, cannulation time, total attempts for catheterization, first, second, and third attempt success, arterial puncture, hematoma, bleeding, and catheter malposition were recorded. The overlap between the carotid artery and IJV in zero- and 45-degree angulation was estimated through ultrasound print. After all, Kolmogorov-Smirnov test was used to assess normal distribution of the data. Then, the data were analyzed through Student's t-test, Mann-Whitney U test, and chi-square test. $\mathrm{P}<0.05$ was considered as statistically significant. Results: The results showed no significant differences between the two groups regarding the duration of different catheterization steps $(\mathrm{P}=0.376)$. In all the cases in both groups, accessing the vein was successful with three attempts or less. There were no clinical complications of catheterization in the two groups. The mean of overlap was 23.60 \pm 33.47 in zero-degree angulation between the head and trunk and $32.72 \pm 36.38$ in 45-degree angulation and this difference was statistically significant $(\mathrm{P}=0.001)$.

Conclusions: The results of the present study showed that both US-guided techniques under investigation had the same duration in different catheterization steps, total success rate, and primary mechanical complications, and could be used in clinics.
\end{abstract}

Implication for health policy/practice/research/medical education:

Application of a new ultrasound technique for internal jugular vein catheterization with fewer complications would be useful. This research is almost the first statistical investigation of the medial-oblique view for internal jugular vein catetherization.

\section{Background}

Central venous catheters are used for hemodynamic

*Corresponding author: Mohammad Mehdi Derakhshan, Shiraz Anesthesiology and Critical Care Research Center, Research Tower, Khalili Ave., Mollasadra St., Shiraz, Iran, Tel/Fax: +98-7136281506,

E-mail:mmderakh@gmail.com monitoring, volume monitoring, drug injection, long-term Total Parentral Nutrition (TPN), accessing alternative kidney therapies, and cardio-pulmonary resuscitation in case of difficult access to peripheral veins. Despite its prevalent usage, the rate of complications resulting from 
using catheters, including arterial puncture, pneumothorax, air embolism, catheter embolism, and cardiac arrhythmia, range from $5 \%$ to $19 \%$ (1).

The Internal Jugular Vein(IJV) is located at the anterolateral position of the carotid artery in $92 \%$, about $1 \mathrm{~cm}$ lateral to the carotid artery in $1 \%$, medial to the carotid artery in $2 \%$, and out of the area specified by landmark in $5.5 \%$ of patients (2). Considering these anatomical differences of IJV in different individuals, using landmark method may increase the complications. Also, atherosclerosis and carotid artery plaques in some heart surgery patients and high level of artery puncture and its complications limit the possibility of using anatomical indicators for catheterization of IJV. The Society of Cardiovascular Anesthesiologists (SCA) considered the ultrasound-guided central venous puncture as the technique of choice, with a classification of " $1 \mathrm{~A}$ " (2). According to a study performed by SCA, $67 \%$ of the anesthesiologists did not use ultrasound and only $15 \%$ used ultrasound as a routine (3).

Many investigations have reported that the high level of success in IJV catheterization on one hand and decreased arterial puncture and other complications (hematoma, hemothorax, and pneumothorax) on the other hand are due to utilization of ultrasound. Although some researches have revealed higher rates of successful first attempt and fewer attempts by using ultrasound-guided catheterization, arterial puncture is still common and its rate has been reported up to $4 \%$ in some studies (4).

Overall, US-guided catheterization of IJV is performed through three main techniques, including "short axis, out of plane", "long axis, in plane", and "oblique". A new technique, "medial-oblique", has also been recommended by some researchers (4).

\section{Objectives}

The present study aims to evaluate the rate of success, duration of different catheterization steps, and primary mechanical complications in the US-guided catheterization of the right IJV via medial-oblique technique and also compare this technique to the short-axis technique in openheart surgery patients.

\section{Patients and Methods}

After confirmation of Anesthesiology and Critical Care Research Center of Shiraz University of Medical Sciences, this electronically randomized clinical trial was performed on 80 patients who referred to cardiac operating room of Namazi hospital, Shiraz, Iran from March to July 2014. The patients were selected through census method and written informed consents were obtained from them. Besides, the study protocol conforms to the ethical guidelines of the 1975 Declaration of Helsinki. Block randomization with website and software was done. Accordingly, the patients were randomly divided into two groups of 40, Short Axis Group (SAG) and Medial-Oblique Group (M-OG).

The inclusion criteria of the study were being openheart surgery patient, age above 18 years, and willingness to participate in the research. On the other hand, the exclusion criteria of the study were cannulation site infection, wound, hematoma, emphysema, and surgery, intensive coagulopathy, IJV cannulation during the past 72 hours, recent cervical trauma with movement limitations, anatomical changes in the cervical vessels, and IJV thrombosis.

\subsection{Participants}

Demographic information, including age, sex, height, and weight, were recorded. Patients' monitoring in the operating room was performed by pulse oximeter, Invasive Blood Pressure (IBP), capnography, and D2 and V5 ECG leads. Anesthesia induction was done using Midazolam (0.05 $0.15 \mathrm{mg} / \mathrm{kg}$ ), Sufentanil (0.25 - $2 \mathrm{ug} / \mathrm{kg})$, Thiopental (3 $4 \mathrm{mg} / \mathrm{kg}$ ), and Pancuronium (0.08 - $0.12 \mathrm{mg} / \mathrm{kg})$. Then, the patients were intubated and mechanical ventilation without end-expiratory pressure was started. Afterwards, $10 \mathrm{~mL} / \mathrm{kg}$ of intravenous normal saline was injected to each patient and catheterization was done by an individual with the experience of more than 25 prior ultrasound-guided catheterizations of the central vein using both techniques under study. Before catheterization, the patients were turned into the 30 degree trendelenburg position. The researcher was present behind the head of the patient. After determining the anatomical landmarks, the catheterization site was disinfected and the ultrasound probe was covered by the sterile cover. The "Fuji Film M-Turbo Sonosite, Linear $30 \mathrm{~mm}, 5$ - $15 \mathrm{MHZ}$ probe, Japan" ultrasound probe was placed transversely between the two heads of the sternocleidomastoid muscle in the level of cricoid cartilage, perpendicular to the axis of the carotid artery and IJV first in zero-degree and then in 45-degree angulation between the head and trunk. After that, sonography and printing were done.

\subsection{Technique for 2D Real-Time US-Guided Short-Axis Puncture (2)}

For short-axis technique, patient's head was positioned at zero-degree angulation with his trunk. Then, the ultrasound device was put on the right side of the patient and the ultrasound probe was placed transversely between the two heads of the sternocleidomastoid muscle in the level of cricoid cartilage, perpendicular to the axis of carotid artery and IJV with the non-dominant hand. After that, the shortaxis image of the vein and artery was placed in the middle of the monitor screen and the " $18 \mathrm{G} \times 2-1 / 211-6.35 \mathrm{~cm}$ " needle was gently introduced with 60 -degree angulation from the vertical plane in the middle of the point of the longitudinal axis of the probe under negative pressure (2). The needle was not seen on the monitor screen and the location of the needle's tip was estimated through movement of the surrounding tissues. When the point of the needle pierced the vein and blood was seen in the syringe, catheterization (Arrow $7 \mathrm{fr}$ triple lumen, $20 \mathrm{~cm}$ catheter length, 0.032 inchdiameter spring- wire guide, USA) was performed through Seldinger technique.

\subsection{Technique for 2D Real-Time US-Guided Medial- Oblique Puncture}

For medial-oblique technique, patient's head was tilted to left to 45 degrees between the head and trunk. The ultrasound device was then located on the right side of the 
patient and ultrasound probe was placed transversely between the two heads of the sternocleidomastoid muscle in the level of cricoid cartilage and perpendicular to the axis of the carotid artery and IJV with the non-dominant hand. The short-axis image of the vein and artery was placed in the middle of the monitor screen and the probe was then rotated 30 degrees counterclockwise. The image of the vein and the artery was seen on the monitor screen. The " $18 \mathrm{G} \times 2-1 / 211-6.35$ cm" needle was gently pushed into the skin with 30 -degree angulation from the vertical plane from the proximal part of the width of the probe using in-plane approach toward the right nipple, from medial-cephalad to lateral-caudad, under negative pressure. The entire length of the needle was observed on the monitor screen, being away from the carotid artery. After the needle entered the vein and blood was seen in the syringe, catheterization (Arrow $7 \mathrm{fr}$ triple lumen, $20 \mathrm{~cm}$ catheter length, 0.032 inch-diameter spring-wire guide, USA) was performed through Seldinger technique.

Each movement of the needle or change in its direction to access the vein was considered as an attempt. Venous access time (from inserting the needle into the skin to aspiration of blood in the syringe), guidewire time (from inserting the needle into the skin to crossing the second marker of the guidewire from the beginning of the needle's body), cannulation time (from inserting the needle into the skin to reaching 15 -degree to the skin surface), total attempts for catheterization, total successful first attempts, number of arterial punctures, bleeding, and hematoma at the puncture site were recorded. Proper catheter placement was confirmed by a portable chest x-ray.

The overlap between the carotid artery and IJV in zeroand 45-degree angulation was estimated through ultrasound print and calculating the percentage of overlap using the cross-sectional diameter of the carotid artery and IJV in the following formula (Figure 1):

[\% overlap $=$ overlap distance $/$ Carotid Artery Diameter (CAD)]
All data analyses were performed using the SPSS statistical software (version 19, Chicago, IL). Kolmogorov-Smirnov test was used to test the normality of quantitative variables, such as access time, guidwire time, cannulation time, and age. The variables with and without normal distribution were compared through independent Student's t-test and Mann-Whitney U test, respectively. Besides, chi-square test was employed for comparing two qualitative variables in the study groups. $\mathrm{P}<0.05$ was considered as statistically significant.

\section{Results}

Demographic information of the patients in the two groups has been presented in Table 1. As this table depicts, the two groups were not significantly different in this regard. The catheterization indicators in both groups have been shown in Table 2. According to the results, access time and cannulation time were shorter in Group 2 (M-OG) compared to Group 1 (SAG), while guidewire time was shorter in Group 1 (SAG) than in Group 2 (M-OG); however, the differences were not statistically significant.

The total success rate of catheterization was $100 \%$ in both groups. In $97.5 \%$ of the cases in Group 2 (M-OG) compared to $90 \%$ of those in Group 1 (SAG), accessing the vein was successful in the first two attempts. In all the cases in both groups, accessing the vein was successful with three attempts or less. Comparison of access times, guidewire times, and cannulation times between the two groups has been presented in Figure 2. The results showed no arterial puncture, hematoma, bleeding, and catheter malposition in the two groups, indicating no significant difference between the two groups in this respect $(\mathrm{P} \approx 1)$.

The mean of overlap was $23.60 \pm 33.47$ in zero-degree angulation between the head and trunk and $32.72 \pm 36.38$ in 45-degree angulation, and the difference was statistically significant $(\mathrm{P}=0.001)$.

Figure 1. Internal Jugular Vein Sonography

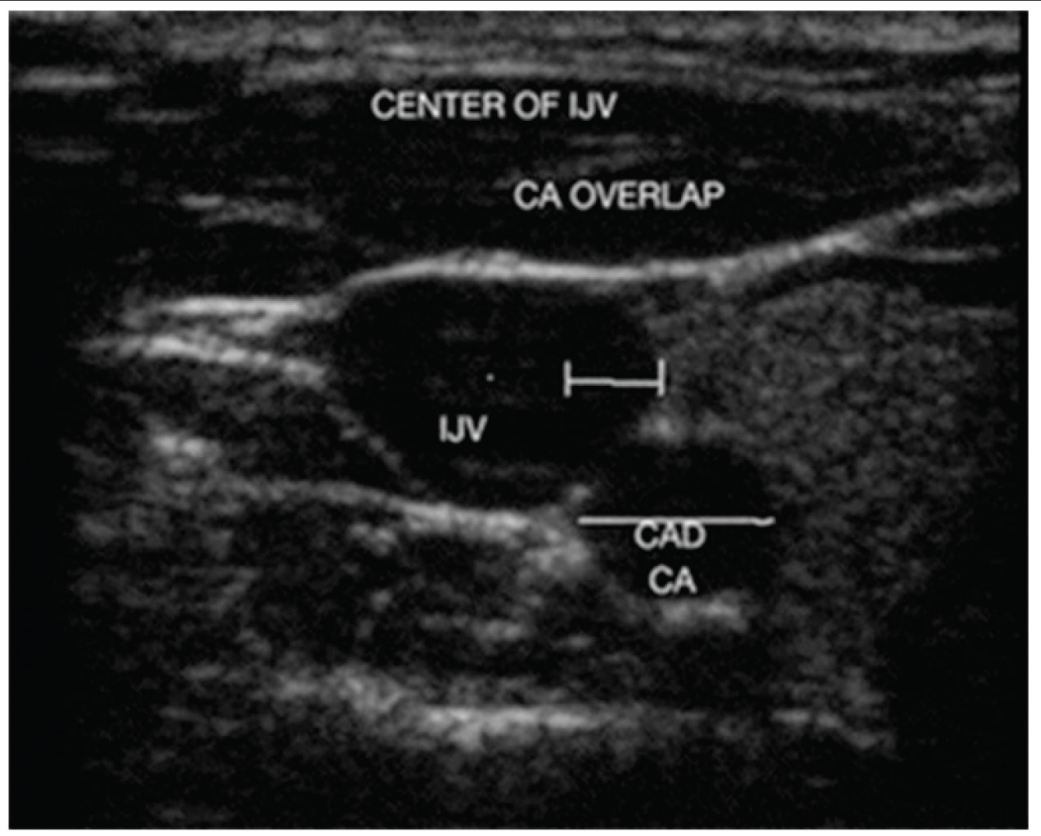

Artery and vein labeled: CA, carotid artery; IJV, internal jugular vein 


\begin{tabular}{|c|c|c|c|c|}
\hline \multirow[t]{2}{*}{ Characteristics } & \multicolumn{4}{|l|}{ Group } \\
\hline & Total $\mathrm{N}=80$ & SAG N $=40$ & MOG N $=40$ & P value \\
\hline Sex & $58.8 / 41.2$ & $62.5 / 37.5$ & $55 / 45$ & 0.496 \\
\hline \multicolumn{5}{|l|}{ M/F (\%) } \\
\hline Age (years) & $59.93 \pm 11.34$ & $59.45 \pm 11.62$ & $60.42 \pm 11.19$ & 0.703 \\
\hline Weight (kg) & $66.23 \pm 9.92$ & $66.62 \pm 10.72$ & $65.85 \pm 9.17$ & 0.729 \\
\hline Height $(\mathrm{cm})$ & $164.81 \pm 7.95$ & $165.97 \pm 8.34$ & $163.65 \pm 7.47$ & 0.182 \\
\hline BMI $(\mathrm{kg} / \mathrm{m} 2)$ & $24.16 \pm 3.45$ & $24.21 \pm 3.52$ & $24.12 \pm 3.41$ & 0.715 \\
\hline
\end{tabular}

The data were reported as mean $\pm \mathrm{SD}$ or number (\%). The difference is significant at $\mathrm{P}<0.05$

\begin{tabular}{lllc}
\hline \multicolumn{4}{l}{ Table 2. Venous Cannulation/Catheterization Characteristics in Group 1 (Short-Axis Group) and Group 2 (Medial-Oblique Group) } \\
\hline & \multicolumn{1}{l}{ Group } & \multicolumn{1}{c}{ P value } \\
\cline { 2 - 3 } Characteristics & SAG N $=\mathbf{4 0}$ & M-OG N = 40 & 0.177 \\
& $17.72 \pm 12.59$ & $14.35 \pm 8.93$ & 0.248 \\
Access time(s) & $31.12 \pm 13.12$ & $36.02 \pm 22.92$ & 0.376 \\
Guidewire time(s) & $98.1 \pm 27.75$ & $93.22 \pm 26.02$ & 0.664 \\
Cannulation time(s) & $1.25 \pm 0.63$ & $1.15 \pm 0.42$ & 0.289 \\
Total attempt count & $34(85)$ & $35(87.5)$ & 0.289 \\
First attempt success rate & $2(5)$ & $4(10)$ & 0.289 \\
Second attempt success rate & $4(10)$ & $1(2.5)$ & 1 \\
Third attempt success rate & $40(100)$ & $40(100)$ & \\
Total success rate &
\end{tabular}

The data were reported as mean $\pm \mathrm{SD}$ or number $(\%)$. The difference is significant at $\mathrm{P}<0.05$

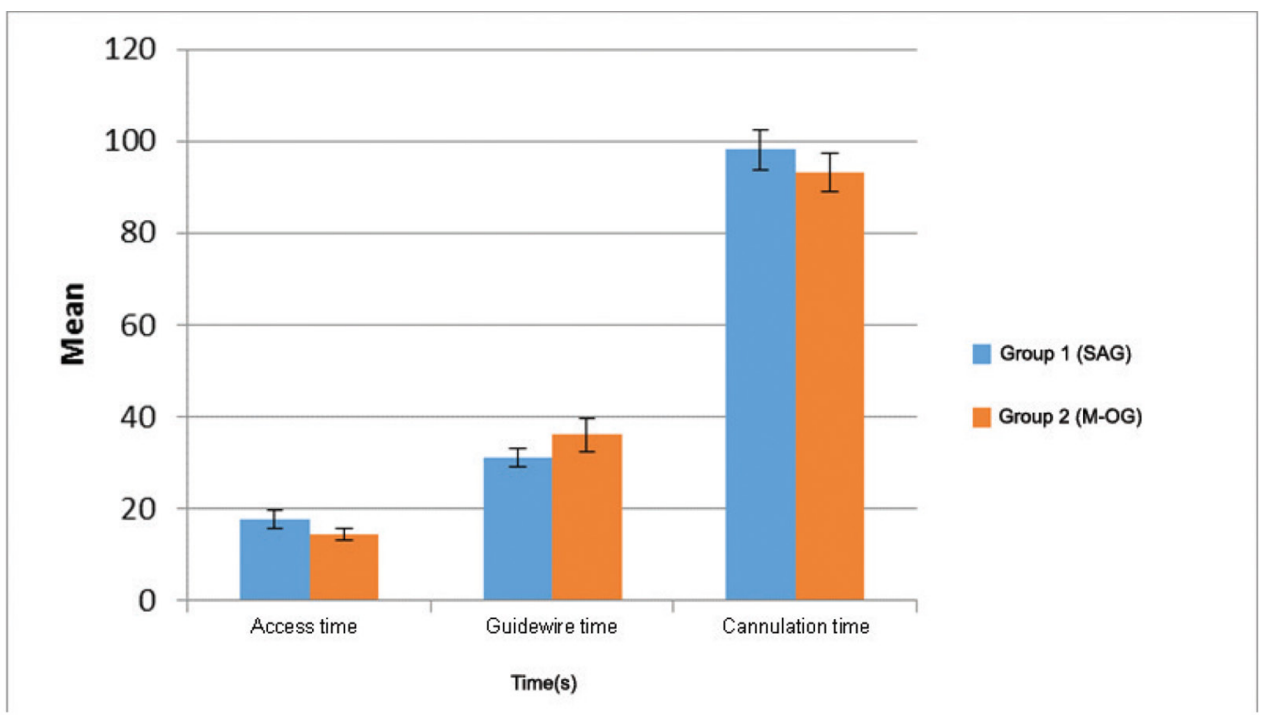

Figure 2. Comparison of Access Time $(\mathrm{P}=0.177)$, Guidewire Time $(\mathrm{P}=0.248)$, and Cannulation Time $(\mathrm{P}=0.376)$ between Group 1 (SAG) and Group 2 (M-OG)

\section{Discussion}

In 1984, ultrasound was first used for central vein cannulation (5). Since then, different techniques have been recommended for ultrasound-guided central vein catheterization due to development of ultrasound portable devices. Vessels can be seen via ultrasound in short-axis (cross-sectional or transverse view), long-axis (longitudinal view), or oblique view. To obtain the short-axis view, the probe is placed at a 90 degree angle to the course of the vein. The vessels appear in cross-section in this view. Before attempting the long-axis view, the clinician should identify the location of the vein using the short-axis view. The probe should then be rotated 90 degrees so that its long axis is parallel to the course of the vein (6). The oblique view is obtained by initially locating the vessel in the short axis followed by rotation of the probe to almost midway between the short-axis and long-axis views. With this technique, both vessels are still visualized on the screen but in a slightly elongated view (7). The advantage of short-axis view is the visibility of the surrounding tissues in relation to the needle, while the benefit of the long-axis view is better visibility of the needle's shaft and tip in its direction and depth. In the oblique view, the image of the needle's shaft and tip is more observable and the surrounding tissues can also be seen (2), but the needle's direction is from lateral to medial. A new technique called medial-oblique has also been recommended by some researchers. In this in-plane technique, the needle's direction is from medialcephalad to lateral-cauded, IJV and carotid artery can be seen besides each other, and the needle's shaft and tip are observable in its entire direction. Therefore, this technique has the benefits of the previous three techniques. 
This research was almost the first statistical investigation of the medial-oblique view and also the first published study comparing short-axis and medial-oblique views. Most studies on short -axis view and its comparison to anatomical landmark-guided technique have not mentioned the duration of different catheterization steps. The access time was 17.72 seconds in Group 1 (SAG) in our study and 12.4 - 240 seconds in other studies $(1,8-11)$. Access time in Group 2 (M-OG) was shorter compared to Group 1, but the difference was not statistically significant.

Guidewire time was longer in Group 2 (M-OG) than in Group 1 (SAG), which can be attributed to the vertical position and greater angle of the needle from the skin surface in Group 2 that cause resistance against advancing the guidewire. However, no statistically significant difference was found between the two groups regarding the guidewire time.

Cannulation time was 98.1 seconds in Group 1 (SAG) in the current study and 58-281 seconds in the previous studies $(9,12,13)$. Additionally, the two study groups were not significantly different in this regard, which might be due to the longer guidewire time in Group 2 (M-OG) in comparison to Group 1 (SAG), causing equal cannulation times in the two groups even with lesser access time in Group 2 (M-OG).

In the present study, all the catheterizations were done with three attempts or less and in less than 180 seconds. In other studies, however, $60 \%$ of the catheterizations were done in less than 180 seconds (14).

Total success rate of catheterization was $100 \%$ in both groups, while it was reported as $93.9-100 \%$ in other studies $(1,15,16)$. Successful catheterization rate in the first attempt was $85 \%$ in Group 1 (SAG) compared to 63 $100 \%$ in other investigations $(8,9,13,15-20)$. In addition, successful catheterization rate in the second attempt was $5 \%$ in Group 1 (SAG) and $2-10 \%$ in other studies (14, 16). Finally, successful catheterization rate in the third attempt was $10 \%$ in Group 1 (SAG), while it was $7.5 \%$ in other studies (14). Overall, the success rates in the first, second, and third attempts in Group 1 (SAG) were similar to the previous studies. Moreover, the results indicated no statistically significant difference between the two groups regarding success rate in the first, second, and third attempts. The success rate in the first two attempts was $90 \%$ in Group 1 (SAG), 87.5 - 100\% in other studies (14, 16), and about $97.5 \%$ in Group 2 (M-OG).

The average number of attempts was 1.25 in Group 1 (SAG) and 1 - 1.5 in other investigations $(8,11,12,16,17,19$, 21). The results also demonstrated no statistically significant difference between the two study groups concerning the mean number of attempts.

Carotid artery puncture is one of the severest complications of jugular vein cannulation. Sometimes, it causes hematoma, airway obstruction, or stroke in patients with carotid artery dissection. The findings of the current study showed no clinical complications of catheterization in Group 1 (SAG), while this measure was $0-3 \%$ in other studies $(1,7-9$, $11-17,19,21)$. The incidence rate of hematoma was also $0 \%$ in this study and $0-4.3 \%$ in other investigations (1, $7,8,11,13,15-17)$. Moreover, there was no statistically significant difference between the two groups with respect to primary mechanical complications. The low incidence rate of major complications in the present study might have resulted from the expertise of the catheterization operator in catheterizing the central vein.

Considering the $4 \%$ rate of arterial puncture in US-guided catheterization of IJV reported in some studies (4), applying a new ultrasound technique with fewer complications would be useful. After introduction of medial-oblique technique, the researchers of this study decided to compare it to short-axis technique. Therefore, the main objective of this study was to compare the success rate, duration of different catheterization steps, and primary mechanical complications of the US-guided catheterization of the right jugular vein using short-axis and medial-oblique techniques among open-heart surgery patients. The study results showed that both US-guided techniques under study had the same duration in different catheterization steps, total success rate, and primary mechanical complications and, consequently, could be used in clinics. Also, using both techniques reduced the major complications compared to the anatomical landmark-guided technique.

Some researchers conducted studies to determine the ideal rotation of the neck for cannulation of IJV. Decreased overlap of the carotid artery and IJV in zero-degree angulation was the result of these investigations. In the present study, a statistically significant difference was found regarding the overlap of the carotid artery and the right IJV in zero-degree and 45-degree angulation between the head and trunk. In our previous study (22), the mean of overlap was $15 \pm 19$ in zero-degree and $27 \pm 26$ in $30-45$ degrees between the head and trunk, which was in line with the findings of the present study. Miki et al. (23) determined the overlap of the carotid artery and the right IJV about 2 $\mathrm{cm}$ and $4 \mathrm{~cm}$ above the clavicle. According to the results, it significantly increased with $>45$ degree rotation of the head in $2 \mathrm{~cm}$ and with $>30$ degree rotation in $4 \mathrm{~cm}$ above the clavicle. In other studies also (24), the overlap varied from $29 \%$ to $44 \%$ in zero-degree and from $42 \%$ to $52 \%$ in 45 degrees. The main reason for using 45-degree rotation of the head to the left while cannulating the right IJV is to see the anatomical landmarks in a better position, but the probability of overlap between the carotid artery and IJV and arterial puncture through its posterior wall may increase. Moreover, in zero-degree angulation of the head, the operator is forced to keep his hand in lateral position and vertically insert the needle into the skin, which increases the probability of sticking the guidewire. Yet, observing the artery and the vein via ultrasound may decrease the possibility of occurrence of major complications. Based on the results of the present study, in cases with no possibility of rotating the neck (such as head and cervical trauma), IJV could be easily cannulated by using ultrasound.

This study had some limitations. Due to the nature of the research, it was impossible to blind the operator to the two techniques under study. One other limitation was that all the study participants were volunteers and there were no children and patients with neck trauma in this study.

\section{Acknowledgements}

This article was extracted from a thesis written by 
Mohammad Mehdi Derakhshan, submitted to School of Medicine in partial fulfillment of the requirements for the fellowship in Cardiac Anesthesiology. This study was funded by the Research Vice-chancellor of Shiraz University of Medical Sciences, Shiraz, Iran (grant No. 92-01-60-7071). Hereby, the authors would like to thank Anesthesiology and Critical Care Research Center of Shiraz University of Medical Sciences for their assistance in publishing this article. Thanks also go to Dr. Nasrin Shokrpour at the Research Consultation Center for improving the use of English in the manuscript.

\section{Authors' Contribution}

Study concept and design: Hamid Kamalipour, Shahrbano Shahbazi; Analysis and interpretation of the data: Mohammad Mehdi Derakhshan; Study supervision: Mohammad Taghi Moinvaziri, Elaheh Allahyari

\section{Financial disclosure}

There is no financial disclosure.

\section{Funding/Support}

This study was financially supported by the Research Vice-chancellor of Shiraz University of Medical Sciences, Shiraz, Iran.

\section{References}

1. MFS Filho LP, de Souza K, Palitot I, Magalhaes I. Comparison between Ultrasound-Guided and Anatomic Landmark Puncture of the Right Internal Jugular Vein. J Cardiovasc Dis Diagn. 2013;1(128):2.

2. Troianos CA, Hartman GS, Glas KE, Skubas NJ, Eberhardt RT, Walker JD, et al. Special articles: guidelines for performing ultrasound guided vascular cannulation: recommendations of the American Society of Echocardiography and the Society Of Cardiovascular Anesthesiologists. Anesth Analg. 2012;114(1):46-72.

3. Ezaru CS, Mangione MP, Oravitz TM, Ibinson JW, Bjerke RJ. Eliminating arterial injury during central venous catheterization using manometry. Anesth Analg. 2009;109(1):130-4.

4. Dilisio R, Mittnacht AJ. The "medial-oblique" approach to ultrasound-guided central venous cannulation--maximize the view, minimize the risk. J Cardiothorac Vasc Anesth. 2012;26(6):982-4.

5. Legler D, Nugent M. Doppler localization of the internal jugular vein facilitates central venous cannulation. Anesthesiology. 1984;60(5):481-2.

6. Mitchell E, Averill L, Wolfson A, Stack A, Eidt J, Mills J. Principles of ultrasound-guided venous access. 2013;UpTODate.

7. Mey U, Glasmacher A, Hahn C, Gorschluter M, Ziske C, Mergelsberg $\mathrm{M}$, et al. Evaluation of an ultrasound-guided technique for central venous access via the internal jugular vein in 493 patients. Support Care Cancer. 2003;11(3):148-55.

8. Airapetian N, Maizel J, Langelle F, Modeliar SS, Karakitsos D, Dupont $\mathrm{H}$, et al. Ultrasound-guided central venous cannulation is superior to quick-look ultrasound and landmark methods among inexperienced operators: a prospective randomized study. Intensive
Care Med. 2013;39(11):1938-44.

9. Leung J, Duffy M, Finckh A. Real-time ultrasonographically-guided internal jugular vein catheterization in the emergency department increases success rates and reduces complications: a randomized, prospective study. Ann Emerg Med. 2006;48(5):540-7.

10. Stone MB, Moon C, Sutijono D, Blaivas M. Needle tip visualization during ultrasound-guided vascular access: short-axis vs long-axis approach. Am J Emerg Med. 2010;28(3):343-7.

11. Tammam TF, El-Shafey EM, Tammam HF. Ultrasound-guided internal jugular vein access: comparison between short axis and long axis techniques. Saudi J Kidney Dis Transpl. 2013;24(4):707-13.

12. Griswold-Theodorson S, Hannan H, Handly N, Pugh B, Fojtik J, Saks $\mathrm{M}$, et al. Improving patient safety with ultrasonography guidance during internal jugular central venous catheter placement by novice practitioners. Simul Healthc. 2009;4(4):212-6.

13. Napolitano M, Malato A, Raffaele F, Palazzolo M, Lo Iacono G, Pinna $\mathrm{R}$, et al. Ultrasonography-guided central venous catheterisation in haematological patients with severe thrombocytopenia. Blood Transfus. 2013;11(4):506-9.

14. Ray BR, Mohan VK, Kashyap L, Shende D, Darlong VM, Pandey RK. Internal jugular vein cannulation: A comparison of three techniques. J Anaesthesiol Clin Pharmacol. 2013;29(3):367-71.

15. Cavanna L, Civardi G, Vallisa D, Di Nunzio C, Cappucciati L, Berte R, et al. Ultrasound-guided central venous catheterization in cancer patients improves the success rate of cannulation and reduces mechanical complications: a prospective observational study of 1,978 consecutive catheterizations. World J Surg Oncol. 2010;8:91.

16. Chittoodan S, Breen D, O'Donnell BD, Iohom G. Long versus short axis ultrasound guided approach for internal jugular vein cannulation: a prospective randomised controlled trial. Med Ultrason. 2011;13(1):21-5.

17. Agarwal A, Singh DK, Singh AP. Ultrasonography: a novel approach to central venous cannulation. Indian J Crit Care Med. 2009;13(4):213-6.

18. Schummer W, Schummer C, Tuppatsch H, Fuchs J, Bloos F, Huttemann E. Ultrasound-guided central venous cannulation: is there a difference between Doppler and B-mode ultrasound? J Clin Anesth. 2006;18(3):167-72.

19. Shrestha BR, Gautam B. Ultrasound versus the landmark technique: a prospective randomized comparative study of internal jugular vein cannulation in an intensive care unit. JNMA J Nepal Med Assoc. 2011;51(182):56-61.

20. Zhang YL, Mi WD, Yu DJ, Fu Q, Feng XX. [Application of ultrasonic surface location for internal jugular vein catheterization via central approach]. Zhongguo Yi Xue Ke Xue Yuan Xue Bao. 2011;33(5):479-84.

21. Turker G, Kaya FN, Gurbet A, Aksu H, Erdogan C, Atlas A. Internal jugular vein cannulation: an ultrasound-guided technique versus a landmark-guided technique. Clinics (Sao Paulo). 2009;64(10):989-92.

22. Bagheri M, Kamalipour H, Javdani M. EF FECTS OF THE VALSALVA MANEUVER AND HEAD ROTATION ON INTERNAL JUGULAR VEIN DIAMETER AND LOCATION BY ULTRASONOGRAPHY. Medical Journal of The Islamic Republic of Iran (MJIRI). 2002;15(4):183-7.

23. Miki I, Murata S, Nakazawa K, Onozawa S, Mine T, Ueda T, et al. Anatomical relationship between the common carotid artery and the internal jugular vein during head rotation. Ultrasound. 2014:1742271X14524571.

24. Wang R, Snoey ER, Clements RC, Hern HG, Price D. Effect of head rotation on vascular anatomy of the neck: an ultrasound study. $J$ Emerg Med. 2006;31(3):283-6. 